\title{
Improved multisensor switching scheme for fault tolerant control
}

\author{
María M. Seron, José A. De Doná and John J. Martínez
}

\begin{abstract}
In this paper we present an improved version of a fault tolerant multisensor switching scheme previously proposed by the authors. The scheme uses multiple sensors-observer pairs and a switching mechanism to automatically detect the healthy components of the system and avoid the use of faulty components in closed loop. The improvements proposed in the current paper include the extension to more general sensor schemes and fault models, and the derivation of a new set of less conservative conditions for fault tolerance guarantees. As a consequence of these improvements, the multisensor scheme can now be used in applications that were not possible before, as illustrated by an example of vehicle lateral control.
\end{abstract}

\section{INTRODUCTION}

We have recently presented a new fault tolerant control (FTC) scheme consisting of multiple sensor-observer pairs and a switching mechanism that implicitly detects the presence of faulty sensors and automatically reconfigures the system to avoid their use in closed loop [1], [2].

A schematic of the multisensor scheme is depicted in Figure 1. Each sensor has an associated state estimator in a structure similar to the dedicated observer scheme of [3]. Each estimator, together with a state feedback gain, is able to individually stabilise the closed loop system. At each instant of time, the switching strategy utilises the state feedback gain to feed back the estimate of the plant states that achieves the best value of a chosen performance criterion.

Performance and stability conditions were analytically explored, allowing the off-line parameterisation of the system to achieve fault tolerance properties. In addition to this implicit FTC property, the scheme proposed in [1], [2] was mainly aimed at maintaining high performance of the system during normal (fault-free) operation. In fact, it has been verified in a number of simulation examples that the switching scheme achieves, in the fault-free case, similar performances to other, more traditional, approaches; e.g., centralised Kalman filter [1] and sensor fusion [2]. Thus, although the switching scheme might seem to discard useful information by selecting only one sensor (or group of sensors) at each time, the performance is not degraded with respect to that of more traditional schemes.

We present here the following improvements of the multisensor switching scheme of [1], [2]:

1) The extension to more general sensor schemes whereby more than one sensor can be associated with each observer;

M.M. Seron and J.A. De Doná are with the Centre for Complex Dynamic Systems and Control, School of Elec. Eng. and Comp. Sci., University of Newcastle. Callaghan 2308, NSW, Australia. De Doná is currently on study leave with a visiting position at CAS, Ecole des Mines de Paris, France.

J.J. Martínez is with the Gipsa-lab UMR 5216, Département Automatique. BP.46, St. Martin D'Hères 38440, France.
2) The derivation of a new set of less conservative conditions for fault tolerance guarantees;

3) The use of tools developed in [4] to refine the construction of the invariant sets employed for the analysis;

4) Allowance for more general fault models, where reduced effectiveness in sensors is also considered.

As a consequence of the reduction of conservatism that results from points 1) to 4) above, the multisensor scheme can now be used in applications that were not possible before; the application example investigated in Section VI is an instance of this.

\section{Multisensor SWitching SCHEME}

In this section, we describe the multisensor switching control scheme depicted in Figure 1.

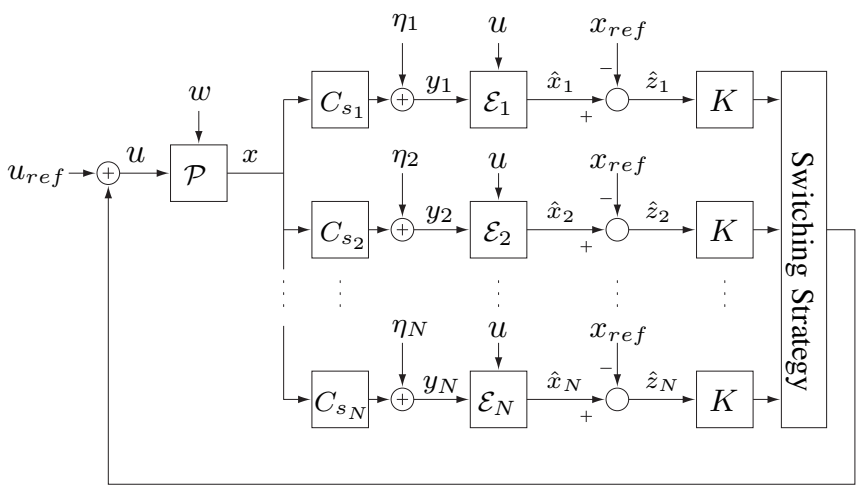

Fig. 1. Multisensor switching scheme with plant $\mathcal{P}$, sensor gains $C_{s_{1}}, \ldots C_{s_{N}}$, estimators $\mathcal{E}_{1}, \ldots, \mathcal{E}_{N}$ and feedback gain $K$

\section{A. Problem statement}

The plant is given by the linear discrete-time model $^{1}$

$$
x^{+}=A x+B u+E w,
$$

where $x \in \mathbb{R}^{n}$ and $x^{+} \in \mathbb{R}^{n}$ are, respectively, the current and successor system states, $u \in \mathbb{R}^{m}$ is the control input and $w \in \mathbb{R}^{r}$ is a bounded process disturbance.

Assumption 2.1 (Disturbance bound): The signal $w \in \mathbb{R}^{r}$ in (1) satisfies ${ }^{2}|w(k)| \leq \bar{w}$ for all discrete-time instants $k \geq$ 0 and some known constant nonnegative vector $\bar{w} \in \mathbb{R}^{r}$. o

The control objective is for the state of the plant (1) to track a signal $x_{r e f}$ generated by the reference model

$$
x_{r e f}^{+}=A x_{r e f}+B u_{r e f} .
$$

Assumption 2.2 (Reference bounds): Constant vectors $x_{r e f, 0} \in \mathbb{R}^{n}$ and $\overline{x_{r e f}} \in \mathbb{R}^{n}, \overline{x_{r e f}} \geq 0$, are known such

\footnotetext{
${ }^{1}$ The time dependency will be omitted when clear from the context.

${ }^{2}$ Inequalities and magnitude of vectors (matrices) are taken elementwise.
} 
that $x_{r e f}(k) \in X_{\text {ref }}=\left\{x \in \mathbb{R}^{n}:\left|x-x_{\text {ref }, 0}\right| \leq \overline{x_{r e f}}\right\}$ for all $k \geq 0$.

\section{B. Sensor Measurements and Fault Model}

We assume that the plant's states are measured via $N$ groups of sensors with outputs $y_{i} \in \mathbb{R}^{p_{i}}, i=1, \ldots, N$. Typically, each group of sensors measures a subset of the components of the state vector, and a particular state variable can be measured by sensors belonging to different groups. It is also possible, under this framework, for a sensor to belong to different groups of sensors; and for some sensors to measure linear combinations of the states. All these situations can be modelled by the following output equations:

$$
y_{i}=\Pi_{i}\left(C_{s i} x+\eta_{i}\right)+\left(I_{p_{i}}-\Pi_{i}\right) \eta_{i}^{F}, \quad i=1, \ldots, N,
$$

where $\eta_{i} \in \mathbb{R}^{p_{i}}$ and $\eta_{i}^{F} \in \mathbb{R}^{p_{i}}$ are bounded measurement disturbances or noises, $I_{p_{i}}$ is the identity matrix of dimension $p_{i} \times p_{i}$ and the pair $\left(A, C_{s_{i}}\right)$ is assumed observable.

Assumption 2.3 (Noise bounds): Bounding sets $\Delta_{i} \triangleq$ $\left\{\eta \in \mathbb{R}^{p_{i}}:|\eta| \leq \overline{\eta_{i}}\right\}$ and $\Delta_{i}^{F} \triangleq\left\{\eta \in \mathbb{R}^{p_{i}}:|\eta| \leq \overline{\eta_{i}^{F}}\right\}$, where $\overline{\eta_{i}} \in \mathbb{R}^{p_{i}}, \bar{\eta}_{i}^{F} \in \mathbb{R}^{p_{i}}, i=1, \ldots, N$, are constant nonnegative vectors, are known such that the noises satisfy $\eta_{i}(k) \in \Delta_{i}$ and $\eta_{i}^{F}(k) \in \Delta_{i}^{F}$, for all $k \geq 0$.

Our fault model is encapsulated in the fault matrix $\Pi_{i} \in$ $\mathbb{R}^{p_{i} \times p_{i}}$, and is described in the following assumption.

Assumption 2.4 (Fault model): We consider abrupt faults that result in an instantaneous change in the fault matrix $\Pi_{i}$ of the sensor output equations ( 3 ) from $\Pi_{i}=I_{p_{i}}$ (all sensors in the $i$ th group are operational) to $\Pi_{i}=\operatorname{diag}\left\{\pi_{i 1}, \ldots, \pi_{i p_{i}}\right\}$, where $\pi_{i j} \in[0,1]$ for $j=1, \ldots, p_{i}$. In particular, $\left(\pi_{i j}=1\right) /$ $\left(0<\pi_{i j}<1\right) /\left(\pi_{i j}=0\right)$ denotes that the $j$ th sensor of the $i$ th group is operational/ has reduced its effectiveness/ is out of order. The faults are persistent, that is, no diagonal entry of $\Pi_{i}$ is allowed to have more than one change in time. $\circ$

The above sensor measurement scheme and fault model extend the framework of [1], [2], where all sensors were assumed to measure the same combination of plant states and only sensor outage was considered.

\section{Estimators}

For each group of sensors with measurements given by (3) we construct full-order state estimators described by

$$
\hat{x}_{i}^{+}=A \hat{x}_{i}+B u+L_{i}\left(y_{i}-C_{s_{i}} \hat{x}_{i}\right), \quad i=1, \cdots, N .
$$

Assumption 2.5: The gains $L_{i}$ are such that the matrices

$$
A_{L_{i}} \triangleq A-L_{i} C_{s_{i}}, \quad i=1, \cdots, N
$$

have eigenvalues strictly inside the unit circle. (This is always possible since $\left(A, C_{s i}\right)$ are assumed observable.)

Remark 2.6: Notice that when all sensors are operational, the estimation errors, defined as

$$
\tilde{x}_{i} \triangleq x-\hat{x}_{i}, \quad i=1, \ldots, N
$$

satisfy, using (1), (3) with $\Pi_{i}=I_{p_{i}}$, (4) and (5),

$$
\tilde{x}_{i}^{+}=A_{L_{i}} \tilde{x}_{i}+E w-L_{i} \eta_{i} .
$$

Hence, using Assumption 2.5, the bounds on the disturbances and noises described in Assumptions 2.1 and 2.3, respectively, and Theorem 3.2 of [2], we can compute the following "ultimate-bound" invariant sets in which the trajectories of each subsystem (7) will remain if started inside or towards which the trajectories will asymptotically converge if started outside:

$$
\begin{aligned}
\tilde{S}_{i} \triangleq\left\{\tilde{x}_{i} \in \mathbb{R}^{n}:\left|V_{i}^{-1} \tilde{x}_{i}\right| \leq H_{i}\right\} \quad \text { where } \\
\\
\quad H_{i} \triangleq\left(I_{n}-\left|\Lambda_{i}\right|\right)^{-1}\left|V_{i}^{-1}\left[\begin{array}{ll}
E & -L_{i}
\end{array}\right]\right|\left[\begin{array}{ll}
\bar{w}^{\prime} & {\overline{\eta_{i}}}^{\prime}
\end{array}\right]^{\prime},
\end{aligned}
$$

for $i=1, \ldots, N$, where $A_{L_{i}}=V_{i} \Lambda_{i} V_{i}^{-1}$ is the Jordan decomposition of $A_{L_{i}}$. From (8), we can compute the following ultimate bound ${ }^{3}$ on $\tilde{x}_{i}$ :

$$
\left|\tilde{x}_{i}\right| \leq \overline{\tilde{x}_{i}} \triangleq\left|V_{i}\right| H_{i}
$$

\section{Switching controller}

To achieve the tracking control objective stated in Section II-A, a controller is employed that switches between sensors-estimator pairs in the following way. At each time step, given the current estimator tracking errors

$$
\hat{z}_{i} \triangleq \hat{x}_{i}-x_{r e f}, \quad i=1, \cdots, N,
$$

the switching controller performs the optimisation

$$
\hat{z}^{*}=\arg \min _{\hat{z}}\left\{\hat{z}^{\prime} P \hat{z}: \hat{z} \in\left\{\hat{z}_{1}, \cdots, \hat{z}_{N}\right\}\right\},
$$

and computes the control action as

$$
u^{*}=u_{r e f}-K \hat{z}^{*},
$$

where $P=P^{\prime} \geq 0$ is a user-selected weighting matrix (which can be chosen to facilitate the satisfaction of the fault tolerant conditions, see Theorem 5.2 in Section $\mathrm{V}$ below) and the gain $K$ is such that the matrix $A-B K$ has all its eigenvalues strictly inside the unit circle. (We will assume that $(A, B)$ is stabilisable, so that this is always possible.)

Thus, at each time instant, the switching controller feeds back the best, with respect to the minimisation (11), estimate tracking error via a linear gain $K$.

\section{ClOSED-LOOP ANALYSIS AND INVARIANT SETS IN THE ABSENCE OF SENSOR FAULT}

Under Assumptions 2.1 to 2.3 and 2.5, if all sensors are operational, the switching control system described in Section II has bounded trajectories regardless of the switching sequence [1], [2]. To see this, consider the plant (1) in closedloop with control $u=u^{*}=u_{\text {ref }}-K \hat{z}^{*}$ given by (12)-(11). Suppose that, at any arbitrary time, the switching controller selects some $l \in\{1, \cdots, N\}$ so that $\hat{z}^{*}=\hat{z}_{l}$ and

$$
u=u^{*}=u_{r e f}-K \hat{z}_{l} .
$$

\footnotetext{
${ }^{3}$ If $\nu(k) \in \mathbb{R}^{m}$ is a discrete-time signal and $\bar{\nu} \geq 0$ is a vector in $\mathbb{R}^{m}$, the notation $|\nu| \leq \bar{\nu}$ denotes the elementwise ultimate bound $|\nu(k)| \leq \bar{\nu}$ for all times $k \geq k^{*}$, for some $k^{*} \geq 0$.
} 
Substituting (13) into (1) and using (2), (10) and (6), we have that the plant tracking error $z \triangleq x-x_{\text {ref }}$ satisfies

$$
z^{+}=(A-B K) z+\left[\begin{array}{ll}
E & B K
\end{array}\right]\left[\begin{array}{ll}
w^{\prime} & \tilde{x}_{l}^{\prime}
\end{array}\right]^{\prime} .
$$

In addition, from (10) and (6) we have that the estimator tracking errors satisfy

$$
\hat{z}_{i}=z-\tilde{x}_{i}, \quad i=1, \ldots, N .
$$

Since $A-B K$ has all its eigenvalues strictly inside the unit circle and $w, \tilde{x}_{i}$ and $\eta_{i}$ are bounded for all $i \in\{1, \ldots, N\}$ (see Remark 2.6) it follows from (14) and (15) that the tracking error $z$ and the estimator tracking errors $\hat{z}_{i}, i=1, \ldots, N$, are bounded. Moreover, they asymptotically converge to zero in the absence of process and measurement disturbances since the same is true for the estimation errors (6) [See (7)].

Remark 3.1: Using Theorem 3.2 of [2], we can compute the following ultimate-bound invariant set in which the trajectories of the tracking error (14) will remain if started inside or towards which the trajectories will asymptotically converge if started outside:

$$
Z \triangleq\left\{z \in \mathbb{R}^{n}:\left|V^{-1} z\right| \leq\left(I_{n}-|\Lambda|\right)^{-1} v\right\},
$$

where $V \Lambda V^{-1}$ is the Jordan decomposition of $A-B K$, and

$$
v \triangleq \max _{l \in\{1, \ldots, N\}}\left|V^{-1}\left[\begin{array}{ll}
E & B K
\end{array}\right]\right|\left[\begin{array}{ll}
\bar{w}^{\prime} & \tilde{\tilde{x}}_{l}^{\prime}
\end{array}\right]^{\prime},
$$

(the maximum is taken elementwise) with $\bar{w}$ and $\overline{\tilde{x}_{l}}$ as defined in Assumption 2.1 and (9), respectively. From (16), we can compute the following ultimate bound on $z$ :

$$
|z| \leq \bar{z} \triangleq|V|\left(I_{n}-|\Lambda|\right)^{-1} v
$$

Remark 3.2: The ultimate-bound invariant sets of the form (8) and (16) are constructed using techniques developed in [5]. A refinement of those techniques was presented in [4], where a procedure was developed to obtain arbitrarily close approximations of the minimal robust positively invariant (mRPI) set for a stable system driven by bounded disturbances. The procedure "shrinks" invariant sets of the form (8) and (16) to obtain refined sets and tighter elementwise bounds that improve (9) and (17). In the sequel, when we refer to the sets (8) and (16) (and any other invariant set to be derived), and associated elementwise bounds (9) and (17), we will consider that, if needed, the procedure of [4] may have already been applied to refine the results.

\section{Closed-loop Analysis and Invariant Sets UNDER SENSOR FAULT}

Consider the dynamics of the estimator tracking errors $\hat{z}_{i}$ in closed loop with (13) and assume that the selected $l$ th estimator corresponds to a group of operational sensors. Substituting (13) into (4), and using (10), (2)-(3), (15), yields

$$
\begin{aligned}
\hat{z}_{i}^{+}=\hat{x}_{i}^{+}-x_{r e f}^{+}= & A_{L_{i}} \hat{z}_{i}+\left(L_{i} \Pi_{i} C_{s_{i}}-B K\right) z+B K \tilde{x}_{l} \\
& +L_{i}\left(\Pi_{i}-I_{p_{i}}\right) C_{s_{i}} x_{r e f}+L_{i} \eta_{i}^{\pi}\left(\Pi_{i}\right)
\end{aligned}
$$

which can be written compactly as

$$
\hat{z}_{i}^{+}=A_{L_{i}} \hat{z}_{i}+B_{i}\left(\Pi_{i}\right) \nu_{l i}\left(\Pi_{i}\right) .
$$

In (18) and (19) we have used (5) and the definitions

$\eta_{i}^{\pi}\left(\Pi_{i}\right) \triangleq \Pi_{i} \eta_{i}+\left(I_{p_{i}}-\Pi_{i}\right) \eta_{i}^{F}$

$B_{i}\left(\Pi_{i}\right) \triangleq\left[L_{i} \Pi_{i} C_{s_{i}}-B K \quad B K \quad L_{i}\left(\Pi_{i}-I_{p_{i}}\right) C_{s_{i}} \quad L_{i}\right]$

$\nu_{l i}\left(\Pi_{i}\right) \triangleq\left[\begin{array}{llll}z^{\prime} & \tilde{x}_{l}^{\prime} & x_{r e f}^{\prime} & \left(\eta_{i}^{\pi}\left(\Pi_{i}\right)\right)^{\prime}\end{array}\right]^{\prime}$.

Let

$$
\overline{\nu_{l i}\left(\Pi_{i}\right)} \triangleq\left[\begin{array}{llll}
\bar{z}^{\prime} & {\overline{x_{l}}}^{\prime} \quad \overline{x_{r e f}} & \overline{\eta_{i}^{\pi}\left(\Pi_{i}\right)}
\end{array}\right]^{\prime} \in \mathbb{R}^{3 n+p_{i}},
$$

where $\bar{z}$ and $\overline{\tilde{x}_{l}}$ were defined in (17) and (9), respectively (see also Remark 3.2), $\overline{x_{r e f}}$ is as in Assumption 2.2 and $\overline{\eta_{i}^{\pi}\left(\Pi_{i}\right)} \triangleq \Pi_{i} \overline{\eta_{i}}+\left(I_{p_{i}}-\Pi_{i}\right) \overline{\eta_{i}^{F}}$ (see Assumption 2.3). Define

$$
\begin{aligned}
& \tilde{v}_{i}\left(\Pi_{i}\right) \triangleq \max _{l \in\{1, \ldots, N\}}\left|V_{i}^{-1} B_{i}\left(\Pi_{i}\right)\right| \overline{\nu_{l i}\left(\Pi_{i}\right)}, \\
& c_{i}\left(\Pi_{i}\right) \triangleq\left(I_{n}-A_{L_{i}}\right)^{-1} L_{i}\left(\Pi_{i}-I_{p_{i}}\right) C_{s_{i}} x_{r e f, 0},
\end{aligned}
$$

where $x_{r e f, 0}$ is as in Assumption 2.2.

To each estimator tracking error subsystem (19) we associate the following invariant sets (see Theorem 3.2 of [2]) parameterised by the fault matrix $\Pi_{i}$ :

$$
\begin{gathered}
\hat{S}_{i}^{F}\left(\Pi_{i}\right) \triangleq\left\{\hat{z}_{i} \in \mathbb{R}^{n}:\left|V_{i}^{-1} \hat{z}_{i}\right| \leq\left(I-\left|\Lambda_{i}\right|\right)^{-1} \tilde{v}_{i}\left(\Pi_{i}\right)+\epsilon\right\} \\
\oplus\left\{c_{i}\left(\Pi_{i}\right)\right\},
\end{gathered}
$$

where $\epsilon \in \mathbb{R}^{n}$ is any nonnegative vector and $\oplus$ denotes Minkowski sum. In particular, when all sensors of the $i$ th group are healthy, then $\Pi_{i}=I_{p_{i}}$ and the associated set is

$$
\hat{S}_{i} \triangleq \hat{S}_{i}^{F}\left(I_{p_{i}}\right)
$$

Note that the sets $\hat{S}_{i}^{F}\left(\Pi_{i}\right)$ are "centred" at $c_{i}\left(\Pi_{i}\right)$; in particular $\hat{S}_{i}$ is centred at 0 since $c_{i}\left(I_{p_{i}}\right)=0$.

Remark 4.1: In the absence of sensor fault, the trajectories of all tracking error subsystems (19)-(22) (with $\Pi_{i}=I_{p_{i}}$ for all $i=1, \ldots, N)$ will enter and remain in the corresponding invariant sets $\hat{S}_{i}$ defined in (27) [5]. When a sensor (group) fails, provided the switching scheme continues to select estimators associated to healthy groups of sensors whose estimation errors satisfy (9), then the trajectories of all "healthy" tracking errors that have already entered the corresponding invariant sets $\hat{S}_{i}$ will remain in these sets [2].

At a fault appearance in the $j$ th group of sensors with associated fault matrix $\Pi_{j}$, the estimator tracking error dynamics will be driven by equation (19). If there is no further change in the matrix $\Pi_{j}$, then the latter dynamics will converge to and then remain in the corresponding invariant set $\hat{S}_{j}^{F}\left(\Pi_{j}\right)$ defined in (26) [5].

In addition, we are interested in obtaining a set that describes the transition of the estimator tracking error dynamics from a healthy to a faulty condition; that is, between $\hat{S}_{j}$ and $\hat{S}_{j}^{F}\left(\Pi_{j}\right)$. Assuming that at the time of the fault the initial state $\hat{z}_{j}$ is in the set (27), then a set characterisation of the first step of (19) after the fault is

$$
\hat{z}_{j}^{+} \in \hat{S}_{j}^{+}\left(\Pi_{j}\right)
$$


where

$$
\begin{aligned}
\hat{S}_{j}^{+}\left(\Pi_{j}\right)= & A_{L_{j}} \hat{S}_{j} \oplus \Xi_{j}\left(\Pi_{j}\right), \\
\Xi_{j}\left(\Pi_{j}\right) \triangleq & \left(L_{j} \Pi_{j} C_{s_{j}}-B K\right) Z \oplus B K \tilde{S} \oplus \\
& \quad L_{j}\left(\Pi_{j}-I_{p_{j}}\right) C_{s_{j}} X_{r e f} \oplus L_{j} \Delta_{j}\left(\Pi_{j}\right),
\end{aligned}
$$

and where $Z$ is as in (16), $\tilde{S} \triangleq$ convex.hull $\left\{\tilde{S}_{1}, \ldots, \tilde{S}_{N}\right\}$, with $\tilde{S}_{i}$, for $i=1, \ldots, N$, defined in (8), $X_{\text {ref }}$ is as in Assumption 2.2 and (see Assumption 2.3)

$$
\Delta_{j}\left(\Pi_{j}\right) \triangleq \Pi_{j} \Delta_{j} \oplus\left(I_{p_{j}}-\Pi_{j}\right) \Delta_{j}^{F} .
$$

Using the results in Section 3.3 of [4], we will construct an arbitrarily close approximation $\Psi_{j}\left(\Pi_{j}\right)$ of the mRPI set for system (19) and such that it satisfies $\hat{S}_{j}^{+}\left(\Pi_{j}\right) \subset \Psi_{j}\left(\Pi_{j}\right)$ (where $\hat{S}_{j}^{+}\left(\Pi_{j}\right)$ is the "after-fault initial-condition set" defined in (29)). Consider the set $\hat{S}_{j}^{F}\left(\Pi_{j}\right)$ defined in (26), where $\epsilon$ is selected such that $\hat{S}_{j}^{+}\left(\Pi_{j}\right) \subset \hat{S}_{j}^{F}\left(\Pi_{j}\right)$, and let

$$
\Psi_{j, 0}\left(\Pi_{j}\right)=\hat{S}_{j}^{F}\left(\Pi_{j}\right) .
$$

Then any set of the sequence

$\Psi_{j, s+1}\left(\Pi_{j}\right)=$ convex.hull $\left\{\hat{S}_{j}^{+}\left(\Pi_{j}\right), A_{L_{j}} \Psi_{j, s}\left(\Pi_{j}\right) \oplus \Xi_{j}\left(\Pi_{j}\right)\right\}$

where $\Xi_{j}\left(\Pi_{j}\right)$ is defined in (30), satisfies $\Psi_{j, s+1}\left(\Pi_{j}\right) \subset$ $\Psi_{j, s}\left(\Pi_{j}\right)$ and is a positively invariant set for (19) which contains the set $\hat{S}_{j}^{+}\left(\Pi_{j}\right)$ (see [4]). We can thus take $\Psi_{j}\left(\Pi_{j}\right)=$ $\Psi_{j, s}\left(\Pi_{j}\right)$ for some value of $s \geq 0$ to approximate the mRPI set for system (19) that contains $\hat{S}_{j}^{+}\left(\Pi_{j}\right)$.

We will next use the above invariant sets to derive conditions which ensure that the optimisation (11) employed by the switching controller always favours a healthy sensor group over any sensor group containing faulty sensors.

\section{Conditions for Healthy Sensor Selection and Closed-loop Stability under Sensor Fault}

We consider the following assumption.

Assumption 5.1 (Fault scenario):

1) At any time instant, either (a) all groups of sensors are operational; or (b) at least one $i$ th group of sensors is operational and has the states of the corresponding estimator tracking error subsystem (19)-(22) (with $\Pi_{i}=I_{p_{i}}$ ) in the invariant set $\hat{S}_{i}$ in (27), and all operational groups of sensors have estimation errors inside the invariant sets $\tilde{S}_{i}$ defined in (8).

2) At the time a $j$ th group of sensors fails with fault matrix $\Pi_{j}, j \in\{1, \ldots, N\}$, the states of the corresponding estimator tracking error subsystem (19)-(22) belong to the invariant set $\hat{S}_{j}$ defined in (27).

We then have the following result.

Theorem 5.2 (Fault Tolerance): Suppose that the sets (29) and (26), $j=1, \ldots, N$, satisfy

$$
\hat{S}_{j}^{+}\left(\Pi_{j}\right) \subset \hat{S}_{j}^{F}\left(\Pi_{j}\right),
$$

and for some value of $s \geq 0$ the set $\Psi_{j}\left(\Pi_{j}\right) \triangleq \Psi_{j, s}\left(\Pi_{j}\right)$ constructed using the recursion (32)-(33) satisfies

$$
\min _{\hat{z} \in \Psi_{j}\left(\Pi_{j}\right)} \hat{z}^{\prime} P \hat{z}>\max _{i=1, \ldots, N, i \neq j}\left\{\max _{\hat{z} \in \hat{S}_{i}} \hat{z}^{\prime} P \hat{z}\right\},
$$

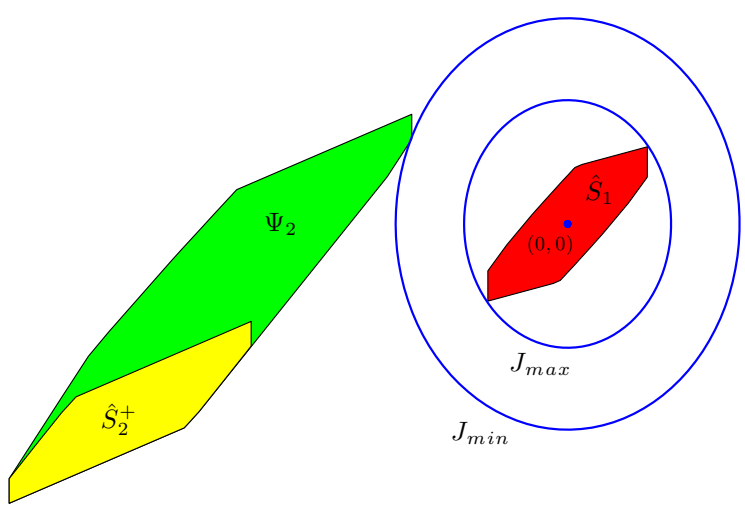

Fig. 2. Illustration of condition (35) of Theorem 5.2 for $N=2$.

where $\hat{S}_{i}$, for $i=1, \ldots, N$, are defined in (27). Then, under Assumption 5.1, the multisensor switching scheme preserves closed-loop stability whenever the $j$ th group of sensors fails with fault matrix $\Pi_{j}$.

Proof: Consider a fault in the $j$ th group of sensors with fault matrix $\Pi_{j}, j \in\{1, \ldots, N\}$, at (continuous-time) $t_{F} \in(k-1, k]$, where $k-1$ and $k$ are two successive discretetime instants. Note that all dynamic states (i.e., (4), (14) and (19)) at time $k$ depend on discrete-time measurements and decisions made at time $k-1$ and are hence not affected by the fault. Thus, any decision made by the switching controller (11)-(12) at time $k$ preserves all state variables within their respective sets and bounds. From Assumption 5.1-(2) we have that the state $\hat{z}_{j}(k)$ of the "faulty" tracking error subsystem (19) is in the set $\hat{S}_{j}$ and hence a set characterisation of the first step $\hat{z}_{j}=\hat{z}_{j}(k) \longrightarrow \hat{z}_{j}^{+}=\hat{z}_{j}(k+1)$ of (19) after the fault is given by (28)-(30). It then follows from the results in Section 3.3 of [4] that the set $\Psi_{j}\left(\Pi_{j}\right)=\Psi_{j, s}\left(\Pi_{j}\right)$ used in condition (35), where $\Psi_{j, s}\left(\Pi_{j}\right), s \geq 0$, satisfies the recursion (33), contains the "after-fault initial-condition set" $\hat{S}_{j}^{+}\left(\Pi_{j}\right)$ and is a positively invariant set for (19) after the fault. Thus, at time $k+1$ and subsequent discrete-time steps, condition (35) and the fact that, by Assumption 5.1(1), at least one operational $i$ th group of sensors satisfies $\hat{z}_{i} \in$ $\hat{S}_{i}$, guarantee that the switching controller (11)-(12) avoids selecting the faulty $j$ th group of sensors and selects, instead, the minimiser (11) amongst healthy groups of sensors, all of which have estimation errors inside the invariant sets $\tilde{S}_{i}$ defined in (8). Note that, again, the latter ensures that all state variables remain within their respective sets and bounds and thus the dynamics on which we have based our analysis (notice, in particular, that (14) and (19)-(22) are driven by $\tilde{x}_{l}$ ) continue to hold. The result then follows.

Figure 2 illustrates condition (35) of Theorem 5.2 for the case $N=2$ groups of sensors, and a fault in the second group of sensors $(j=2)$. In this figure, we have used the notation $J_{\text {min }} \triangleq \min _{\hat{z} \in \Psi_{2}} \hat{z}^{\prime} P \hat{z}$ and $J_{\text {max }} \triangleq \max _{\hat{z} \in \hat{S}_{1}} \hat{z}^{\prime} P \hat{z}$ and the argument ' $\left(\Pi_{2}\right)$ ' has been omitted for clarity. It is evident from the figure that condition (35) can be geometrically interpreted as the separation of the sets $\hat{S}_{1}$ and $\Psi_{2}$ by a level surface of $\hat{z}^{\prime} P \hat{z}$. Note also that, by construction (see (33)) $\Psi_{2}$ contains the "after-fault initial-condition set" $\hat{S}_{2}^{+}$. 


\section{A. Comparison with the methodology of [2], [4]}

The switching controller proposed ${ }^{4}$ in [2] was based upon updated estimate tracking errors, defined as

$$
\hat{z}_{i}^{U P} \triangleq \hat{z}_{i}+M_{i}\left(y_{i}-C_{s_{i}} \hat{x}_{i}\right), \quad \text { for } i=1, \ldots, N,
$$

where $\hat{z}_{i}$ is as in (4)-(10), $M_{i}$ satisfies $A M_{i}=L_{i}$ and $L_{i}, \hat{x}_{i}$ are as in (4). Then, instead of (11), the controller performed the optimisation

$\hat{z}^{U P *}=\arg \min _{\hat{z}^{U P}}\left\{\left(\hat{z}^{U P}\right)^{\prime} P \hat{z}^{U P}: \hat{z}^{U P} \in\left\{\hat{z}_{1}^{U P}, \cdots, \hat{z}_{N}^{U P}\right\}\right\}$,

and computed the control action as $u^{*}=u_{r e f}-K \hat{z}^{U P *}$. In accordance with the latter controller, the fault tolerance conditions (34)-(35) of Theorem 5.2 were replaced in [2] by

$$
\hat{S}_{j} \subset \hat{S}_{j}^{F}\left(\Pi_{j}\right),
$$

where $\hat{S}_{j}$ is as in (27) and $\hat{S}_{j}^{F}\left(\Pi_{j}\right)$ is as in (26), and

$$
J_{j}^{\text {min }}>\max _{i}\left\{J_{i}^{\max }: i \in\{1, \ldots, N\}, i \neq j\right\},
$$

where $J_{j}^{\min } \triangleq \min _{\hat{z}^{U P} \in S_{j}^{\min }\left(\Pi_{j}\right)}\left(\hat{z}^{U P}\right)^{\prime} P \hat{z}^{U P}, J_{i}^{\max } \triangleq$ $\max _{\hat{z} U P \in S_{i}^{\max }}\left(\hat{z}^{U P}\right)^{\prime} P \hat{z}^{U P}$, and

$$
\begin{array}{r}
S_{j}^{\text {min }}\left(\Pi_{j}\right) \triangleq( \\
\left(I_{n}-M_{j} C_{s_{j}}\right) \hat{S}_{j}^{F}\left(\Pi_{j}\right) \oplus M_{j} \Pi_{j} C_{s_{j}} Z \oplus \\
M_{j}\left(\Pi_{j}-I_{p_{j}}\right) C_{s_{j}} X_{r e f} \oplus M_{j} \Delta_{j}\left(\Pi_{j}\right), \\
S_{i}^{\text {max } \triangleq}\left(I_{n}-M_{i} C_{s_{i}}\right) \hat{S}_{i} \oplus M_{i} C_{s_{i}} Z \oplus M_{i} \Delta_{i},
\end{array}
$$

and where $Z$ is defined in (16), $X_{\text {ref }}$ is as in Assumption 2.2, $\Delta_{j}\left(\Pi_{j}\right)$ is defined in (31) and $\Delta_{i}$ is as in Assumption 2.3. In addition, the techniques of [4] can be used to tighten the sets involved in (37)-(40) and further relax the assumptions on the parameters of the system (reference bounds, noise characteristics, etc.) necessary to satisfy the above conditions.

We will next qualitatively compare conditions (37)-(38) of [2] with conditions (34)-(35) of the present paper. Due to the inclusion condition (37), both the first summand in the definition of the set (39) and the first summand in the definition of the set (40) contain the origin (recall that the sets $\hat{S}_{i}$ are centred at zero). Thus, the "separation" of the sets (39) and (40) — necessary to satisfy condition (38) heavily relies on large values of the reference "offset" $x_{r e f, 0}$ (see Assumption 2.2) which, in turn, offsets the reference set $X_{\text {ref }}$ and, hence, $S_{j}^{\min }\left(\Pi_{j}\right)$ in (39), away from zero. However, the larger the offset $x_{r e f, 0}$ is, the farther from the origin the "centre" $c_{j}\left(\Pi_{j}\right)$ of $\hat{S}_{j}^{F}\left(\Pi_{j}\right)$ is (see (25)). Thus, since $\hat{S}_{j}$ does not "shift" with the reference offset, the value of $\epsilon$ in (26) necessary to satisfy the inclusion condition (37) may be relatively large. This may result in a "large" set $\hat{S}_{j}^{F}\left(\Pi_{j}\right)$, which may obstruct the desired separation.

On the other hand, although the separation of the sets (27) and (29)-required to satisfy the new condition (35) also relies on the reference offset $x_{r e f, 0}$, note that increasing the value of $x_{r e f, 0}$ also tends to aid the inclusion

\footnotetext{
${ }^{4}$ The overview presented here is a slight generalisation of the techniques of [2] in the sense that we allow for a fault matrix $\Pi_{j}$ as in Assumption 2.4, whereas [2] only considered the case of sensor outage $\left(\Pi_{j}=0\right)$.
}

condition (34), since both sets $\hat{S}_{j}^{+}\left(\Pi_{j}\right)$ (see (29)-(30)) and $\hat{S}_{j}^{F}\left(\Pi_{j}\right)$ (see (26)-(25)) shift with the reference offset.

Our experience with several examples confirms the above analysis and, furthermore, suggests that the offset values needed to satisfy condition (35) tend to be much smaller than those required to satisfy (38). Moreover, in some applications it may even be impossible to satisfy condition (38) with realistic values for the system parameters. This fact is illustrated in the example of Section VI.

\section{Vi. Application to Vehicle Lateral Control}

We consider the problem of vehicle lateral control, which consists of controlling an autonomous vehicle's lateral movements, with its longitudinal speed constant, to either keep the vehicle on the centre of the road or to change lanes. We shall focus on the vision-based lateral control scheme described in [6], where one or more video cameras are employed to obtain measurements of the offset of the vehicle with respect to the lane at a certain distance ahead of the vehicle, called the "lookahead" distance (see also [7]).

A linearised model of the vehicle's lateral and yaw dynamics is typically used for lateral controller design. This model assumes small steering angles and a linear tire model, and is parameterised by the current longitudinal velocity. Coupling the front wheels and the rear wheels together, the resulting bicycle model is described by the following equations [6]:

$\left[\begin{array}{c}\dot{v}_{y} \\ \ddot{\psi}\end{array}\right]=\left[\begin{array}{cc}-\frac{c_{f}+c_{r}}{m v_{x}} & \frac{c_{r} l_{r}-c_{f} l_{f}}{m v_{x}}-v_{x} \\ \frac{-l_{f} c_{f}+l_{r} c_{r}}{I_{\psi} v_{x}} & -\frac{l_{f}^{2} c_{f}+l_{r}^{2} c_{r}}{I_{\psi} v_{x}}\end{array}\right]\left[\begin{array}{c}v_{y} \\ \dot{\psi}\end{array}\right]+\left[\begin{array}{c}\frac{c_{f}}{m} \\ \frac{l_{f} c_{f}}{I_{\psi}}\end{array}\right] \delta_{f}$,

where $v_{x}, v_{y}$ are the longitudinal and lateral velocity, respectively, $\dot{\psi}$ is the yaw rate, $\delta_{f}$ is the front wheel steering angle, $m$ is the total mass of the vehicle, $I_{\psi}$ is the yaw moment of inertia, $l_{f}, l_{r}$ are the distances to the front and rear axles, respectively, from the centre of gravity, and $c_{f}, c_{r}$ are the cornering stiffness of the front and rear tires.

The vision system has a lookahead distance, $L$, and provides two additional measurements: $y_{L}$, the offset from the right side of the road at the lookahead, and $\epsilon_{L}$, the angle between the tangent of the road and the vehicle orientation. The equations of evolution of these measurements due to the motion of the car and changes in the road geometry are [6] $\dot{y}_{L}=v_{x} \epsilon_{L}-v_{y}-\dot{\psi} L, \dot{\epsilon}_{L}=v_{x} K_{L}-\dot{\psi}$, where $K_{L}$ represents the curvature of the road. We combine this vision dynamics with the vehicle lateral dynamics (41) into a single model with state $x=\left[\begin{array}{llll}y_{L} & \epsilon_{L} & v_{y} & \dot{\psi}\end{array}\right]^{\prime}$, control input $u=\delta_{f}$, disturbance input $w=K_{L}$, and output $y=\left[\begin{array}{lll}y_{L} & \epsilon_{L} & \dot{\psi}\end{array}\right]^{\prime}$. Using $L=10 \mathrm{~m}$ and the parameter values (from [7]) $v_{x}=20 \mathrm{~m} / \mathrm{s}$, $m=1569 \mathrm{~kg}, I_{\psi}=272.4 \mathrm{Ns} / \mathrm{rad}, l_{f}=1.35 \mathrm{~m}, l_{r}=1.37 \mathrm{~m}, c_{f}=$ $59600 \mathrm{~N} / \mathrm{rad}$ and $c_{r}=86600 \mathrm{~N} / \mathrm{rad}$, and sampling the resulting model at $0.1 \mathrm{~s}$, the vehicle lateral discrete dynamics can be described by (1) with

$$
A=\left[\begin{array}{cccc}
1 & 2 & -0.1684 & -0.1803 \\
0 & 1 & -0.0087 & -0.0173 \\
0 & 0 & 0.4949 & -0.2172 \\
0 & 0 & 0.0811 & -0.0268
\end{array}\right], B=\left[\begin{array}{c}
-4.8428 \\
-0.4609 \\
-4.1003 \\
5.4545
\end{array}\right], E=\left[\begin{array}{l}
2 \\
2 \\
0 \\
0
\end{array}\right] \text {. }
$$

The road curvature is assumed to be a random signal bounded as $w \leq \bar{w}=0.0001$. 


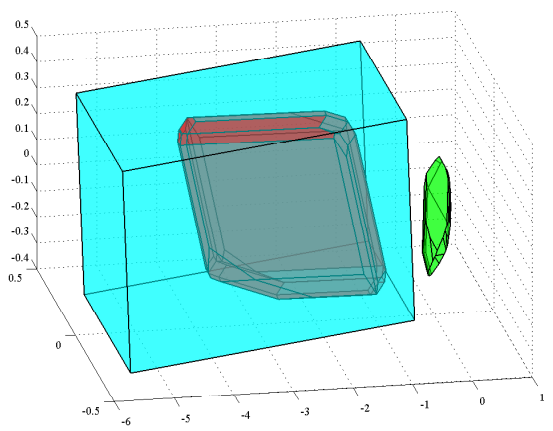

Fig. 3. Sets for the example, cut through $v_{y}=0.3$.

We consider a lane change maneuver, which is accomplished by supplying a reference trajectory to the lateral control system [6], [7]. The reference signal is designed so that the vehicle travels a full lane width (assumed here to be $3 \mathrm{~m}$ ) along the lateral axis with respect to its body orientation within a finite time period (taken here as $7 \mathrm{~s}$ ) and aligns itself with the adjacent lane at the end of the maneuver. The reference signal bound parameters (Assumption 2.2) are $x_{r e f, 0}=\left[\begin{array}{llll}3 & 0 & 0 & 0\end{array}\right]^{\prime}, \overline{x_{r e f}}=\left[\begin{array}{llll}1.5 & 0.06 & 0.06 & 0.06\end{array}\right]^{\prime}$. We consider two identical groups of sensors having outputs (3) with matrices and noise bounds (Assumption 2.3): $C_{s i}=\left[\begin{array}{llll}1 & 0 & 0 & 0 \\ 0 & 1 & 0 & 0 \\ 0 & 0 & 0 & 1\end{array}\right], \overline{\eta_{i}}=\left[\begin{array}{c}0.01 \\ 0.001 \\ 0.001\end{array}\right], \overline{\eta_{i}^{F}}=\overline{\eta_{i}}, i=1,2$. The estimator gains in (4) are computed by pole placement [to place the eigenvalues of $A-L_{i} C_{s i}$ at $\left.(0.1,0.2,0.3,0.4)\right]$ as $L_{i}=$ $\left[\begin{array}{llll}0.8643 & 2.0084 & -0.2827 \\ 0.0077 & 0.7004 & -0.0250\end{array}\right], i$

$\left[\begin{array}{ccc}0.0077 & 0.7004 & -0.0250 \\ -0.1156 & -0.0068 & -0.0691\end{array}\right], i=1,2$. The switching matrix in $\left[\begin{array}{rrr}0.061156 & -0.0068 & -0.0691 \\ -0.0240 & -0.0039 & -0.0966\end{array}\right]$

(11) is selected as $P=\operatorname{diag}\{1,0,0,0\}$ and the gain in (12) is computed as $K=\left[\begin{array}{llll}0.0248 & 0.2620 & -0.0210 & 0.0525\end{array}\right]$ to place the eigenvalues of $A-B K$ at $(0.5,0.6,0.7,0.8)$. We consider the fault-matrix $\Pi_{1}=\Pi_{2}=\Pi=\operatorname{diag}\{0,0,1\}$ for either group of sensors. Note that this represents the total loss of the measurements $y_{L}, \epsilon_{L}$ from either vision system.

We compute $\hat{S}_{1}^{+}\left(\Pi_{1}\right)=\hat{S}_{2}^{+}\left(\Pi_{2}\right)$ as defined in (29) and $\hat{S}_{1}=\hat{S}_{2}$ as defined in (27) (and the intermediate sets required for their computation). Taking $\epsilon=$ $\left[\begin{array}{llll}0.5630 & 0.5456 & 0.2818 & 0.0033\end{array}\right]^{\prime}$, the set $\hat{S}_{1}^{F}\left(\Pi_{1}\right)=$ $\hat{S}_{2}^{F}\left(\Pi_{2}\right)$ defined in (26) satisfies condition (34). In addition, condition (35) is satisfied with left hand side equal to 0.8040 and right hand side equal to 0.2854 . Applying Theorem 5.2 we can conclude that the switching controller will preserve stability under the considered fault type. On the other hand, choosing $\epsilon=\left[\begin{array}{llll}1.5853 & 1.9707 & 0.1302 & 0.8652\end{array}\right]^{\prime}$ to satisfy condition (37), the separation condition (38) does not hold for the above system parameters (the left hand side of (38) equals 0.2650 whereas the right hand side equals 1.2684). Thus, fault tolerance of the scheme could not be guaranteed using the results of [2].

Figure 3 shows the sets for this example in the 3D space defined by (the tracking errors for) $\left(y_{L}, \epsilon_{L}, \dot{\psi}\right)$, cut through $v_{y}=0.3$. The large set (in blue) is $\hat{S}_{1}^{F}\left(\Pi_{1}\right)=\hat{S}_{2}^{F}\left(\Pi_{2}\right)$, the (red) set contained inside it is $\hat{S}_{1}^{+}\left(\Pi_{1}\right)=\hat{S}_{2}^{+}\left(\Pi_{2}\right)$, and the small (green) set on the right hand side is $\hat{S}_{1}=\hat{S}_{2}$.

We simulated the above lateral control system under the switching control strategy proposed in this paper over 90s.
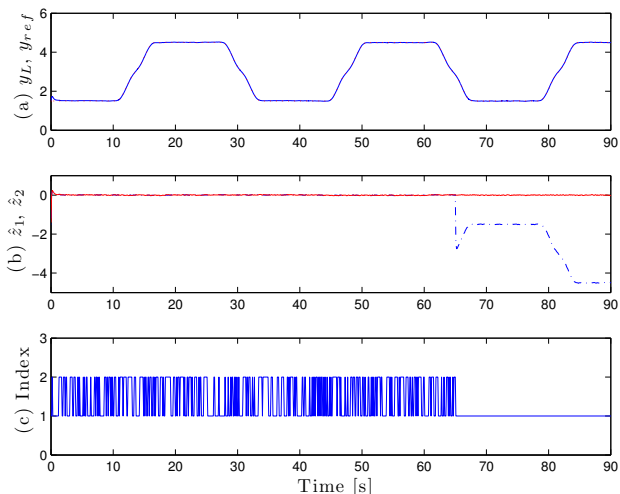

Fig. 4. Lateral control simulation under the proposed scheme.

Both group of sensors start healthy and at $65 \mathrm{~s}$ a fault with matrix $\Pi_{2}$ occurs in the second group of sensors. The resulting responses are shown in Figure 4. Plot (a) shows the vehicle lateral position $y_{L}$ (blue solid) together with its reference signal (red dotted); note that both signals are almost indistinguishable. Plot (b) shows the 1st component of the tracking errors $\hat{z}_{1}$ (red) and $\hat{z}_{2}$ (blue, dash-dot) of the first and second group of sensors-observer, respectively; notice the jump in $\hat{z}_{2}$ due to the fault at $65 \mathrm{~s}$. Plot (c) shows the index of the observer selected at each sampling time by the switching mechanism. Note that the sequence oscillates between both observers before the occurrence of the fault and then correctly selects observer 1 afterwards.

\section{CONCLUSIONS}

We have presented an improved version of a previously proposed fault tolerant multisensor switching scheme. The scheme uses multiple sensors-observer pairs and a switching mechanism that automatically avoids the use of faulty components in closed loop. The improvements proposed here include the extension to more general sensor schemes and fault models, and the derivation of a new set of less conservative conditions for fault tolerance guarantees. As a consequence of these improvements, the multisensor scheme can now be used in applications that were not possible before, as illustrated by the presented example.

\section{REFERENCES}

[1] J. Martínez, X. Zhuo, J. De Doná, and M. Seron, "Multisensor switching strategy for automotive longitudinal control," in Proc. of the 2006 American Control Conference, Minneapolis, MN, 2006.

[2] M. Seron, X. Zhuo, J. De Doná, and J. Martínez, "Multisensor switching control strategy with fault tolerance guarantees," Automatica, vol. 44, no. 1, pp. 88-97, 2008.

[3] R. Clark, "Instrument fault detection," IEEE Transactions on Aerospace and Electronic Systems, vol. 14, pp. 456-465, 1978.

[4] S. Olaru, J. De Doná, and M. Seron, "Positive invariant sets for fault tolerant multisensor control schemes," in Proceedings 17 IFAC World Congress, Seoul, Korea, July 2008.

[5] E. Kofman, H. Haimovich, and M. Seron, "A systematic method to obtain ultimate bounds for perturbed systems," International Journal of Control, vol. 80, no. 2, pp. 167-178, February 2007.

[6] C. Taylor, J. Košecká, R. Blasi, and J. Malik, "A comparative study of vision-based lateral control strategies for autonomous highway driving," International J. of Robotics Research, vol. 18, no. 5, pp. 442-453, 1999.

[7] C. Hatipoglu, U. Özgüner, and K. Redmill, "Automated lane change controller design," IEEE Transactions on Intelligent Transportation Systems, vol. 4, no. 1, pp. 13-22, 2003. 